\title{
An automatic blood vessel segmentation method for retinal images
}

\author{
Jingdan Zhang ${ }^{1,}$, Le Wang ${ }^{1, b}$, Yingjie Cui ${ }^{1, c}$, Wuhan Jiang ${ }^{2, d}$ \\ ${ }^{1}$ Department of Electronics and Communication, Shenzhen Institute of Information Technology, \\ Shenzhen 518172, China \\ ${ }^{2}$ Fada Road, Longgang District, Shenzhen 518129, China \\ azhangjd358@163.com, ${ }^{\mathrm{b}}$ wangle@sziit.com.cn, ${ }^{\mathrm{c}}$ cuiyj@sziit.com.cn, ${ }^{\mathrm{a}}$ whjiang_1114@163.com
}

\begin{abstract}
Keywords: medical image segmentation, retinal images, graph-based algorithm, K-mean algorithm.

Abstract. This paper presents an automatic blood vessel segmentation method for retinal images. Our method integrates the dual-tree complex wavelet transform (DT-CWT) with the graph-based algorithm. The DT-CWT is used to construct the multi-scale features for each pixel, which is directionally selective and robust to noise. The graph-based algorithm is exploited to classify each pixel as vessel or non-vessel. Our method is validated on the publicly available DRIVE database, and compared with the state-of-the-art algorithms.
\end{abstract}

\section{Introduction}

Diabetic retinopathy is a complication of diabetes and is a major cause of blindness in developed countries. Blood vessel segmentation from retinal images plays a crucial role for diagnosing complications due to hypertension, diabetes, arteriosclerosis, cardiovascular disease and stroke [1]. Automatic blood vessel segmentation system could reduce the doctors' workload, and increase the effectiveness of preventive protocols and early therapeutic treatments.

Many approaches have been reported in the area of blood vessel segmentation of retinal images, including rule-based method [3], model-based method [4-6], matched filtering [7-9], and supervised method [2][10-13]. However, the retinal images have low contrast, and large variability is presented in the image acquisition process [2], which deteriorate automatic blood vessel segmentation results.

In this paper, we partition the retinal image into two types: vessel and non-vessel. Effective feature presentation could improve the segmentation results. We integrate the dual-tree complex wavelet feature with original intensity as the pixel feature vector, which is robust to shift and noise variation. Then, a graph-based method [14] is exploited to segment the retinal images into multiple vessel regions and non-vessel regions. Finally, we label the regions with K-mean method for final classification.

The rest of this paper is organized as follow. Section 2 describes our proposed method. In Section 3 , experimental results are presented, followed by the conclusion in Section 4.

\section{Our proposed method}

Feature Extraction: In original RGB retinal images, the green channel shows the best vessel-background contrast, while the red and blue channels show low contrast and are noisy. So, we select the green channel from the RGB retinal image, and the green channel intensity of each pixel is taken as the intensity feature. Fig. 1 (a) is an original RGB retinal image from DRIVE database, and its green channel image is shown in Fig.1 (b).

Retinal images often show important lighting variations, poor contrast and noise [2]. Wavelet theory provides a powerful framework to decompose images into different scales and orientations. Dual-tree complex wavelet transform (DT-CWT) has been developed by Kingsburg [15], which is a particularly suitable tool for feature extraction, as it is directionally selective, approximately shift invariant, limited redundant, and robust to noise. We create DT-CWT feature from the high-frequency sub-bands of DT-CWT with scale three and four. DT-CWT high-frequency images with scale three and four of Fig.1(b) are shown in Fig.1 (c) and (d). 

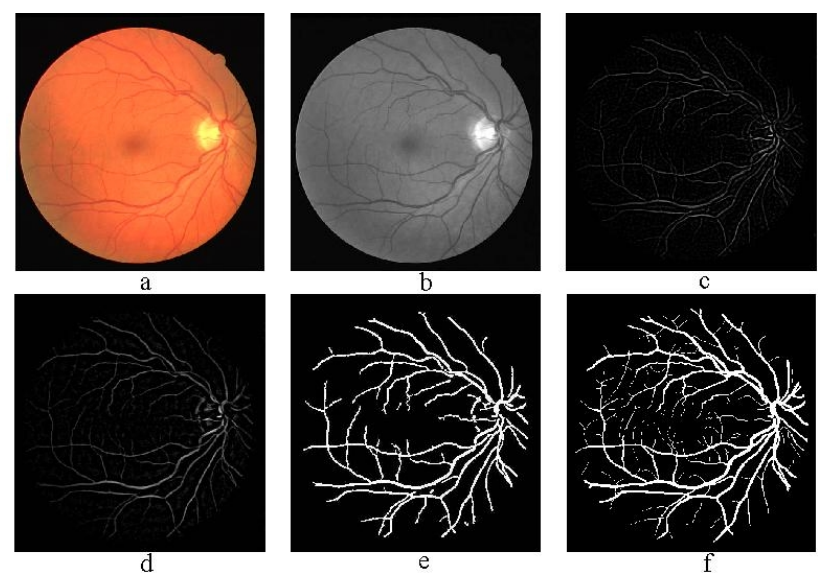

Fig.1 Illustration of the feature extraction process. (a) Original RGB retinal image. (b) The green channel of the original image. (c) The high-frequency image of DT-CWT with scale three. (d) The high-frequency image of DT-CWT with scale four. (e) The segmentation result with our proposed method. (f) The manual segmentation result by the first specialist.

Graph-based segmentation method: Felzenszwalb [14] proposed an efficient graph-based images segmentation method. An important characteristic of this method is its ability to preserve detail in low variability image regions which ignoring details in high variability regions. Retinal images have lighting variations and poor contrast. Therefore, we exploit Felzenszwalb's method for blood vessel segmentation could overcome the problem of lighting variations and poor contrast in retinal images. Moreover, this method is highly efficient, running in time nearly linear in the number of image pixels. So, this method can be used in the medical processing application.

Let $G=(V, E)$ be an undirected graph with vertices $v_{i} \in V$, the set of elements to be segmented, and edges $\left(v_{i}, v_{j}\right) \in E$ corresponding to pairs of neighboring vertices. Each edge $\left(v_{i}, v_{j}\right) \in E$ has a corresponding weight $w\left(v_{i}, v_{j}\right)$, which is a non-negative measure of the dissimilarity between neighboring elements $v_{i}$ and $v_{j}$. In the case of retinal image segmentation, the elements in $V$ are pixels and weight of the edge $\left(v_{i}, v_{j}\right)$ is the difference in feature vector between the pixels $v_{i}$ and $v_{j}$ $\left(w\left(v_{i}, v_{j}\right)=\left|f_{v_{i}}-f_{v_{j}}\right|\right)$.

In the graph-based method, a segmentation $S$ is a partition of $V$ into components such that each component (or region) $C \in S$ corresponds to a connected component in a graph $G^{\prime}=\left(V, E^{\prime}\right)$, where $E^{\prime} \in E$. We want the elements in a component to be similar and elements in different component to be dissimilar.

Felzenszwalb [14] defines inter difference of a component $C \subseteq V$ to be the largest weight in the minimum spanning tree of the component, $M S T(C, E)$. That is, $\operatorname{Int}(C)=\max _{e \in M S T(C, E)} w(e)$. One intuition underlying this measure is that a given component $C$ only remains connected when edges of weight at least $\operatorname{Int}(C)$ are considered. Then, he defines the difference between two components $C_{1}, C_{2} \subseteq V$ to be the minimum weight edge connecting the two components. That is, $\operatorname{Dif}\left(C_{1}, C_{2}\right)=\min _{v_{i} \in C_{1}, v_{j} \in C_{2},\left(v_{i}, v_{j}\right) \in E} w\left(v_{i}, v_{j}\right)$. If there is no edge connecting $C_{1}$ and $C_{2}$, we let $\operatorname{dif}\left(C_{1}, C_{2}\right)=\infty$. Then, Felzerszwalb [14] defines the pairwise comparison predicate as

$$
D\left(C_{1}, C_{2}\right)=\left\{\begin{array}{l}
\text { true if } \operatorname{Dif}\left(C_{1}, C_{2}\right)>\operatorname{MInt}\left(C_{1}, C_{2}\right), \\
\text { false otherwise }
\end{array}\right.
$$

where the minimum internal difference, MInt is defined as, $\operatorname{MInt}\left(C_{1}, C_{2}\right)=\min \left(\operatorname{Int}\left(C_{1}\right)+\tau\left(C_{1}\right), \operatorname{Int}\left(C_{2}\right)+\tau\left(C_{2}\right)\right)$. The threshold function $\tau$ is defined as $\tau(C)=k /|C|$, where $|C|$ denotes the size of $C$, and $k$ is some constants parameter.

In our work, we exploit Felzenswalb's graph-based method [14] to partition the retinal image. Vessels of the retinal image belong to the detail information. To reserve the thin and small vessels in the 
segmentation result, $k$ is not a larger value. So, there are multiple regions (vessel region or non-vessel regions) after the graph-based segmentation.

Postprocessing: After clustering with the graph-based algorithm, there are multiple regions including vessel regions and non-vessel regions. We use the mean intensity feature of pixels in a region to estimate the region class. K-mean method is used to label the region class based on the regions' inherent distance from each other. Finally, in the visual inspection, small isolated regions misclassified as blood vessels are also observed. If the vessel region is connected with no more than 30 pixels, it will be reclassified as non-vessel. The segmentation result of our proposed method on Fig.1 (a) is shown in Fig.1 (e).

\section{Experimental results}

Database and similarity indice: The DRIVE database [12] is used in our experiments. This dataset is a public retinal image database, and is widely used by other researchers to test their blood vessel segmentation methods. Moreover, the DRIVE database provides two sets of manual segmentations made by two different observers for performance validation. In our experiments, performance is computed with the segmentation of the first observer as ground truth. To quantify the overlap between the segmentation results and the ground truth for vessel pixels and non-vessel pixels, accuracy (Acc) are adopted in our experiments.

Comparing with other methods: In order to compare our approach with other retinal vessel segmentation algorithms, the average Acc value is used as measures of method performance. We compare our method with the following published methods: Martinez-Parez et al. [3], Jiang and Mojon [4], Chaudhuri et al. [7], Cinsdikici and Aydin [9]. The comparison results are summarized in Table 1, which indicate our proposed method outperforms most of the other methods.

Table 1 Comparing the segmentation results of different algorithms with our method on DRIVE database in terms of average Acc value.

\begin{tabular}{|l|l|l|}
\hline \multicolumn{1}{|c|}{ Method Type } & \multicolumn{1}{c|}{ Method } & \multicolumn{1}{c|}{ DRIVE } \\
\hline \hline Rule-based method & Martinez-Perez et al. , [3] & 0.934 \\
\hline Model-based method & Jiang and Mojon, [4] & 0.891 \\
\hline \multirow{2}{*}{ Matched filter } & Chaudhuri et al. , [7] & 0.877 \\
\cline { 2 - 3 } & Cinsdikici and Aydin, [9] & 0.929 \\
\hline Clustering method & Our proposed method & 0.930 \\
\hline
\end{tabular}

\section{Conclusions}

This study proposes a blood vessel segmentation method based on the graph-based algorithm. To overcome the problem of low contrast and large variability in retinal images, we construct the feature vector with the green channel intensity, and the high-frequency sub-bands of DT-CWT with scale three and four. Then, we partition the retinal image into multiple regions with the graph-based algorithm. Finally, the multiple regions are classified by K-mean algorithm as vessel regions or non-vessel regions. Our method is validated on the DRIVE database with available gold standard images. In addition, we compare our method with the state-of-art methods, and experimental results indicate that out method outperforms most of the other methods.

\section{Acknowledgements}

This project is supported in part by Shenzhen Science and Technology plan Project (JCYJ20120615101059717), and Project of Shenzhen Institute of Information Technology (SYS201004). 


\section{References}

[1] J.J. Kanski. Clinical Ophthalmology: A systematic approach. London: Butterworth-Heinemann, (1989).

[2] S. Roychowdhury, D.D. Koozekanani and K.K. Parhi: IEEE journal of biomedical and health informatics. Vol.99 (2014), DOI: 10.1109/JBHI.2014.2335617.

[3] M.E. Marinez-Perez, A.D. Hughes, S.A. Thom, A.A. Bharath and K.H. Parker: Medical Imaging Anaysis. Vol.11 (2007), p. 47.

[4] X. Jiang and D. Mojon: IEEE Transactions on Pattern Analysis and Machine Intelligence. Vol.25 (1) (2003), p. 131.

[5] K.A. Vermeer, F.M. Vos, H.G. Lemij and A.M. Vossepoel: Computers in Biology and Medicine. Vol.34 (2004), p. 209.

[6] B. Al-Diri, A. Hunter and D. Steel: IEEE Transactions on Medical Imaging. Vol.28 (2009), p. 1488.

[7] S. Chaudhuri, S. Chatterjee, N. katz, M. Nelson and M. Goldbaum: IEEE Transactions on Medical Imaging. Vol.8(3) (1989), p. 263.

[8] J. Odstrcilik, R. Kolar, A. Budai, and et al.: IET image processing, Vol. 7 (2013), p. 373.

[9] M.G. Cinsdikici and D. Aydin: Comput. Methods Programs Biomed. Vol. 96 (2009), p. 85.

[10] D. Marin, A. Aquino, M.E. Gegundez-Arias and J.M. Bravo: IEEE Transactions on medical imaging. Vol. 30 (2011), p. 146.

[11] M. Niemeijer, J. Staal, B.V. Ginneken, M. Loog, M.D. Abramoff, J. Fitzpatrick and M. Sonka: SPIE Med. Imag. Vol. 5370 (2004), p. 648.

[12] J. Staal, M.D. Abramoff, M. Niemeijer, M.A. Viergever and B. Ginneken: IEEE Transactions on Medical Imaging. Vol. 23 (2004), p. 501.

[13] G.B. Kande, T.S. Savithri, and P.V. Subbaiah: International journal of computer science and network security. Vol. 7 (2007), p. 102.

[14] P. Felzenszwalb and D. P. Huttenlocher: International Journal of Computer Vision. Vol. 59 (2004), p. 167.

[15] N. G. Kingsbury: Journal of Applied and Computational Harmonic Analysis. Vol. 10 (2001), p. 234. 